\title{
Desenvolvimento de algoritmos de controle para o uso em quadricópteros com aplicações em enxa- mes de robôs
}

Souza, D. A. B.

Escola Politécnica de Pernambuco Universidade de Pernambuco 50.720-001 - Recife, Brasil dabs@ecomp.poli.br
Feitosa, M. A. F.

Escola Politécnica de Pernambuco

Universidade de Pernambuco

50.720-001 - Recife, Brasil

marcíliofeitosa@uol.com.br

Resumo Este artigo apresenta um design para modelar e construir um protótipo de um UAV quadrotor. Também são propostos alguns dispositivos elétricos e sensores a serem utilizados, mostrando suas características e necessidades no projeto. Para escolha dos dispositivos foram feitas comparações e estudos baseados em outros projetos.

Abstract This paper presents a model to design and build a prototype of a quadrotor UAV. Also are proposed some electric devices and sensors to be used, showing their characteristics and their necessities in the project. To choose the devices were made comparisons based in other projects. 


\section{Introdução}

O estudo de UAV's (veículos aéreos não tripulados do inglês Unmanned aerial vehicles) tem crescido bastante nos últimos anos. O tipo de UAV proposto nesse trabalho é o quadrotor, um equipamento composto por quatro motores e que tem se tornado muito popular devido à sua grande utilidade em diversas aplicações.

$\mathrm{Na}$ configuração de um quadrotor, quatro motores iguais são fixados nas extremidades de uma estrutura em forma de + , e no centro ficam os dispositivos de controle e sensoriamento. Cada motor possui sentido de rotação específico, de modo que motores adjacentes sempre possuem sentido de rotação opostos.

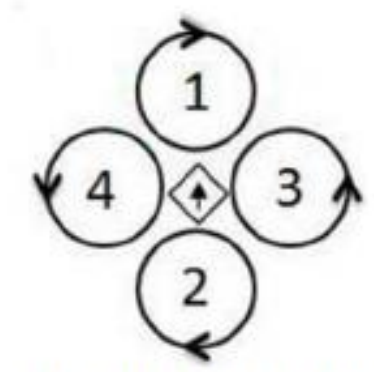

Configuração de um quadrotor

O controle de um quadrotor é feito através do ajuste das velocidades dos seus motores, e os seus movimentos são realizados através de alguns comandos: Throttle (aceleração) que é o movimento vertical, Yaw (guinada) é o movimento de rotação no eixo $\mathrm{Z}$, Roll (rolagem) é o movimento de rotação no eixo X, e Pitch (arfagem) é o movimento de rotação no eixo $\mathrm{Y}$.

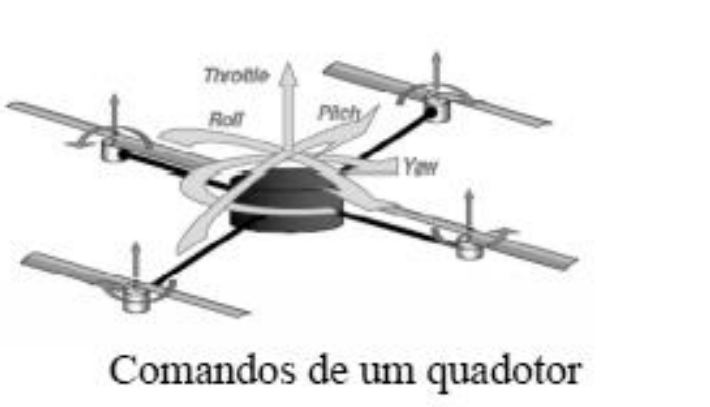

O design de um projeto de um UAV é complexo e exige a integração entre controle e sensoriamento. $\mathrm{Na}$ construção de um quadrotor são necessários: quatro motores; quatro ESCs (Controladores Eletrônicos de Veloci- dade), sendo um para cada motor; uma unidade de processamento, que neste caso é uma placa com microcontrolador; uma unidade de sensoriamento, que possui acelerômetro, giroscópio e altímetro.

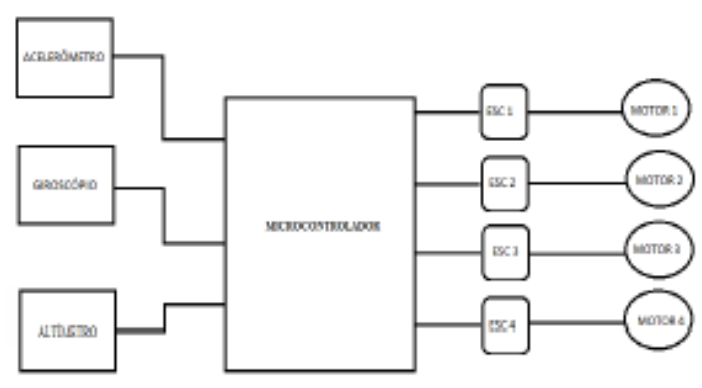

\section{Estrutura de um quadrotor}

\section{Algoritmos de Controle}

Um quadrotor é um sistema que possui 6 graus de liberdade: três deles fazem referência a posição $(x, y, z)$ e os outros três definem a orientação do veículo $(\theta, \beta, \omega)$, que representam roll, pitch e yaw, respectivamente.

A relação entre as coordenadas $(\mathrm{x}, \mathrm{y}, \mathrm{z})$ e os ângulos $(\theta, \beta, \omega)$ formados pelos eixos é de independência. Um movimento através do eixo $\mathrm{x}$, por exemplo, não implica, obrigatoriamente, em uma mudança de qualquer um de seus ângulos.

$\mathrm{O}$ vetor a seguir contem as coordenadas dos três eixos e dos três ângulos de liberdade do quadrotor:

$$
\vec{q}=(\vec{p}, \vec{\alpha})
$$

Onde

$$
\begin{aligned}
\vec{p} & =(x, y, z) \\
\vec{\alpha} & =(\theta, \beta, \omega)
\end{aligned}
$$

Uma maneira de chegar às equações que representam a dinâmica de um quadrotor é utilizando o formalismo de Lagrange. A mecânica Lagrangeana (por possuir um sistema de coordenadas mais geral do que a mecânica newtoniana, por exemplo) consegue resolver problemas mais 
complexos e discrimina fenômenos que podem atingir velocidades relativísticas (velocidades muito altas) com igual precisão daqueles com velocidades mais baixas.

O primeiro passo é definir o langrariano do sistema, que corresponde a soma das energias cinéticas subtraída de toda a energia potencial que atua no sistema.

$$
f=\sum_{i=1}^{N} K i-\sum_{j=1}^{M} U j
$$

onde $\square \square$ corresponde a energia cinética e $\square \square$ a energia potencial.

Em um quadrotor, as energias cinéticas que atuam no sistema correspondem aos movimentos de translação e rotação. Essa energia, segundo [9], é dada por:

$$
\square \square \square \square \square \square=12 \square \square 2
$$

e

$$
\square \square \square \square=12 \square \square 2
$$

onde $\square$ é a primeira derivada de $\square$ e corresponde a velocidade linear, $\square$ corresponde a velocidade angular, $\square$ é a massa e $\square$ corresponde ao momento de inércia do quadrotor.

O momento de inércia de uma partícula é dado por:

$$
\square=\square \square 2
$$

onde $\square$ corresponde a massa e $\square$ à distância em relação ao eixo.

A única energia potencial presente no sistema é a energia potencial gravitacional, que é dada por:

$$
\square=\square \square \square
$$

em que $\square$ é a distância em relação ao chão.

O lagrangeano pode ser então reescrito como:

$$
\mathcal{\delta}=12 \square \square 2+12 \square \square 2-\square \square \square
$$

Finalmente para encontrar as equações de movimento do sistema é necessário resolver a equação de Euler-Lagrange

$\partial / \partial \square \square-\partial / \partial \square \square=\square \square$ onde $\square$ é um vetor de forças e $\square \square$ é késimo componente dessa força. Resolvendo a equação de Euler-Lagrange para cada uma das coordenadas resulta nas seguintes equações:

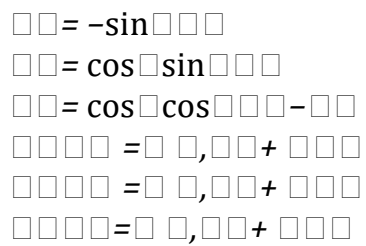

onde Fz corresponde a força produzida por todos os motores, $\square$ é a aceleração no eixo x e assim por diante,; Jyy representa o momento de inércia das rotações através do ângulo $\square$ e assim por diante; $\square \square, \square \square$ representa os termos de Coriolis devido a rotação no eixo z; e $\square \square \square$ corresponde ao torque presente no eixo $\mathrm{x}$ e assim por diante.

Com as equações de movimento definidas, será possível a implementação dos algoritmos de controle que podem ser baseados em diversas técnicas de inteligência artificial. Alguns projetos que utilizavam técnicas de redes neurais e logica fuzzy foram analisados, mas a determinação da técnica a ser utilizada e o início do desenvolvimento dos algoritmos não foi realizados.

\section{Motor}

O motor escolhido foi o do modelo 2822, do fabricante E-max. É um motor do tipo brushless. Este tipo de motor vem sendo bastante utilizado nos últimos anos, esse crescimento se deve ao fato de que a transferência de energia aos seus enrolamentos não depende de um sistema de comutação interno, como nos tradicionais motores de corrente contínua, que geram muito ruído elétrico e desgaste interno. Além disso, esses motores possuem uma melhor relação peso eficiência do que os motores tradicionais.

Para fazer o controle da transferência de energia é necessária a utilização de um controlador de velocidade.

O E-max 2822 possui uma faixa de tensão entre 7,4 e $11,1 \mathrm{~V}$, consumo de corrente entre 7 e $12 \mathrm{~V}$, velocidade máxima de $1200 \mathrm{RPM} / \mathrm{V}$ e pesa 39 gramas.

\section{ESC}

Os Controladores eletrônicos de velocidade ou ESCs (do inglês - Eletronic Speed Controllers) são utilizados para transferir energia e controlar a velocidade dos rotores. No caso de quadrotores cada motor deve ser controlado por um ESC já que as velocidades dos quatro motores não variam de maneira uniforme. 
O ESC mais apropriado foi o E-MAX 18A, pois atende as especificações do motor escolhido. Funciona a uma tensão de $11 \mathrm{~V}$ e pesa 22 gramas. Possui três entradas: duas delas devem estar ligadas a alimentação da bateria e a outra a um sinal PWM.

O sinal de entrada PWM deve ter um período de $20 \mathrm{~ms}$ $(50 \mathrm{~Hz})$ e a largura de pulso deve estar entre $1 \mathrm{~ms}$ (motor desligado) e $2 \mathrm{~ms}$ (motor funcionando a velocidade máxima), permanecendo em nível lógico baixo durante o estante do período.

\section{Plataforma de desenvolvimento}

A plataforma de desenvolvimento é composta pelo microcontrolador que é o cérebro por trás do projeto, será em sua interface que será implementado o programa que controlará a estabilidade de voo do quadricóptero, baseados nas informações recebidas da unidade de sensoriamento.

O microcontrolador ARM Córtex M3 foi definido como dispositivo a ser utilizado no projeto, graças ao seu grande potencial computacional e principalmente devido a sua compatibilidade com as necessidades de visão computacional.

\section{Placa de sensoriamento}

A unidade de sensoriamento é responsável pelas informações como: posição, orientação e aceleração do UAV, que serão utilizadas no controle do voo. É composta por acelerômetro, giroscópio e altímetro.

$\mathrm{O}$ dispositivo tem voltagem de operação entre $1,8 \mathrm{e}$ $3,6 \mathrm{~V}$, consome uma corrente de $10 \mu \mathrm{A}$, possui interface $\mathrm{I}^{2} \mathrm{C}$ e dimensões de $5 \mathrm{~mm} \times 5 \mathrm{~mm} \times 1,2 \mathrm{~mm}$.

\section{Bateria}

O modelo escolhido é o Thunder Power Pro Power 3S 30C $2250 \mathrm{mAh}$, pesa $170 \mathrm{~g}$ e possui um tensão de saída de $11 \mathrm{~V}$, que será ligada em paralelo com os ESC's para alimenta-los, e também alimentará o microcontrolador.

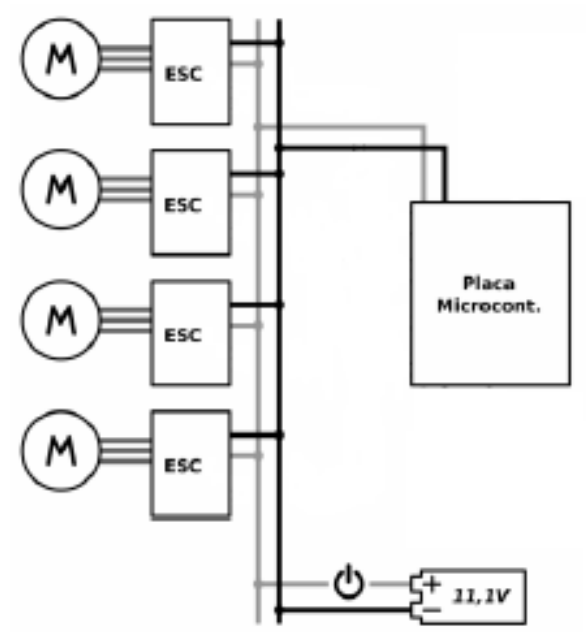

Esquema de ligação da bateria

A bateria definida é do tipo LIPO (do inglês - Lithiumion polymer), este tipo possui uma densidade de carga maior que a das baterias de níquel, oferecendo uma maior duração de carga e um peso menor, características essenciais para projetos deste tipo. Esse tipo de bateria é o mais utilizado na maioria dos projetos analisados.

Estima-se que com a bateria completa o quadricóptero consiga se manter no ar por alguns minutos, não tendo sido feitos testes para poder afirmar por quanto tempo a bateria suporta alimentar o equipamento.

\section{Conclusão}

O design apresentado pode ser usado como base para construção de um quadrotor; A utilização de outros dispositivos pode ser feita de acordo com as necessidades específicas de cada projeto.

\section{Referências}

[1] Alexandre Secchin de Melo. Implementação de um quadrotor como plataforma de desenvolvimento para algoritmos de controle. Master's thesis, Universidade Federal do Espírito Santo, 2010.

[2] Pounds, P., Mahony, R., and Corke, P.,"Modelling and Control of a Quad-Rotor Robot", In Proceedings of the Australasian Conference on Robotics and Automation, 2006 
[3] Rahul Goel, Sapan M. Shah, Nitin K. Gupta, and N. Ananthkrishnan. Modeling, Simulation and Flight Testing of na Autonomous Quadrotor. Proceedings of ICEAE, pages 1-7, 2009.

[4] David Alain, Análise de requisitos de hardware em projeto de UAV quadrotor. Trabalho de conclusão de curso, 2011

[5] David A. do Nascimento, Cristóvão Z. Rufino, Sergio Campello Oliveira, Marcílio André Félix Feitosa, Modeling and Design of an Autonomous Unmanned Aerial Vehicle Quadrotor Prototype for Applications in Swarm of Robots, 2012 